\title{
Predicting postoperative day 1 hematocrit levels after uncomplicated hysterectomy
}

\author{
Carolyn W. Swenson *, Michael S. Lanham, Daniel M. Morgan, Mitchell B. Berger \\ Department of Obstetrics and Gynecology, University of Michigan, Ann Arbor, MI, USA
}

\section{A R T I C L E I N F O}

\section{Article history:}

Received 17 October 2014

Received in revised form 5 January 2015

Accepted 19 March 2015

\section{Keywords:}

Hysterectomy

Postoperative hematocrit

\begin{abstract}
A B S T R A C T
Objective: To develop a model for predicting postoperative hematocrit levels after uncomplicated hysterectomy. Methods: In a retrospective study, data were analyzed from the Michigan Surgery Quality Collaborative for nonemergent hysterectomies performed for benign indications among women aged at least 18 years between January 1, 2012, and April 4, 2014. Linear mixed models were used for univariate and multivariate analyses. Results: The model was developed with data from 4747 hysterectomies and validated on 1184 cases. In the mixed multivariate analysis, higher postoperative day 1 (POD1) hematocrit levels were associated with higher weight ( $B=0.03222, P<0.001$ ), higher preoperative hematocrit $(B=0.6587, P<0.001)$, and non-vaginal hysterectomy $(B=0.2815, P=0.0055)$. Lower POD1 hematocrit was associated with higher preoperative platelet count $(B=-0.00457, P<0.001)$, greater estimated blood loss $(B=-0.00652, P<0.001)$, and larger intraoperative crystalloid volume $(B=-0.3303, P<0.001)$. The final model predicted POD1 hematocrit within $4 \%$ points of the actual value for $91.7 \%$ of cases in the validation set. Conclusion: Use of the model after uncomplicated hysterectomy might help to support the practice of selectively conducting postoperative hematocrit tests after hysterectomy in a clinically thoughtful and cost-effective manner.

(c) 2015 International Federation of Gynecology and Obstetrics. Published by Elsevier Ireland Ltd. All rights reserved.
\end{abstract}

\section{Introduction}

Hysterectomy is the most common major gynecologic surgery performed in the USA, and the second most common surgery performed for women of reproductive age (after cesarean delivery) [1]. Evidencebased guidelines exist for preoperative management, including prophylaxis against surgical-site infections and venous thromboembolism [2]. By contrast, postoperative protocols are largely determined by historical practices and expert opinion rather than by scientific evidence. Although numerous textbooks recommend blood tests-including a complete blood count-as part of the routine postoperative evaluation of a patient, several studies suggest that such routine laboratory testing rarely results in meaningful differences in clinical outcomes [3,4].

Studies have also attempted to identify factors that predict blood counts. One looking at patients undergoing cardiopulmonary bypass surgery [5] identified various factors including older age, female sex, Hispanic or non-white ethnic origins, lower body surface area, and high creatinine as independent predictors of a hematocrit level of $21.9 \%$ or lower. Petersen et al. [6] looked at predictors of postpartum hematocrit after vaginal delivery and found that an estimated blood loss of more than $500 \mathrm{~mL}$, Hispanic ethnic origin, and third- or fourth-

\footnotetext{
* Corresponding author at: University of Michigan, Department of Obstetrics and Gynecology, L4000 Women's Hospital, 1500 E. Medical Center Dr., SPC 5276, Ann Arbor, MI 48109-5276, USA. Tel.: +1 734764 8429; fax: +1 7346479727.

E-mail address: scarolyn@med.umich.edu (C.W. Swenson).
}

degree perineal laceration were objective predictors of a hematocrit level of less than $26 \%$ [6]. The aim of these previous studies was to be able to predict which patients would have hematocrit values at or below a certain threshold; however, few studies have provided a model capable of accurately predicting a postoperative blood count value. To our knowledge, the only published study has been based on orthopedic procedures with a study sample of which less than $40 \%$ were women [7].

Given the lack of evidence supporting routine postoperative laboratory tests after benign gynecologic surgery, the aim of the present study was to develop a mathematical model that can accurately predict postoperative hematocrit level after hysterectomy for benign indications. Such a model might not only limit the number of unindicated procedures that patients undergo after hysterectomy, but also decrease unnecessary resource utilization.

\section{Materials and methods}

In a retrospective study, data were analyzed from the Michigan Surgical Quality Collaborative (MSQC) [8] for hysterectomies performed across the state of Michigan, USA, between January 1, 2012, and April 4, 2014. Informed consent was not required because the MSQC database contains de-identified data. The University of Michigan Institutional Review Board granted "Not Regulated" status to the present study j(HUM00073978). 
The MSQC is an ongoing collaboration involving academic and community hospitals across Michigan and is funded by the Blue Cross Blue Shield of Michigan/Blue Care Network. Trained nurse data abstractors prospectively collate data on patient characteristics, intraoperative processes of care, and 30-day postoperative outcomes, from patients undergoing surgery at participating hospitals.

For the present study, data were reviewed from all women aged 18 years or older who underwent a hysterectomy during the study period at an MSQC member hospital. To model postoperative hematocrit levels after uncomplicated hysterectomies performed for benign indications, only patients with current procedural terminology codes and International Classification of Diseases, Clinical Modification 9 codes consistent with benign, non-emergent hysterectomy for a nonobstetric indication were considered for the analysis. The inclusion criteria were a preoperative hematocrit level greater than or equal to $20 \%$, postoperative day 1 (POD1) hematocrit greater than or equal to $15 \%$, intraoperative crystalloid infusion less than $5 \mathrm{~L}$, no intraoperative colloid infusion, estimated blood loss (EBL) of $10-500 \mathrm{~mL}$, operative duration greater than or equal to 15 minutes, preoperative platelet count greater than or equal to $100000 / \mu \mathrm{L}$, body mass index (calculated as weight in kilograms divided by the square of height in meters) greater than or equal to 15, and an American Society for Anesthesiologists physical status classification of 1 (normal, healthy patient), 2 (mild systemic disease), or 3 (severe systemic disease). Patients were excluded if they had a diagnosis of cancer as a result of the surgery, cancer as an indication for the surgery, preoperative sepsis, other concomitant major surgeries (e.g. colectomy, or liver or kidney surgery), or red-blood-cell or platelet transfusion preoperatively, intraoperatively, or postoperatively.

To build and test the hematocrit prediction model, the study sample was randomly divided into a model development subset and a validation subset, respectively. Modeling and statistical analyses were done via SAS version 9.4 (SAS Institute, Cary, NC, USA) using "proc mixed," "proc reg" with "vif" and "collin" options, "proc anova," and "proc freq" with "chisq" option, as appropriate. Comparisons of continuous and categorical variables between the two subsets were done via one-way analysis of variance and $\chi^{2}$ tests, respectively.

To derive the model, patient demographics and preoperative, intraoperative, and postoperative characteristics associated with blood loss and fluid balance were selected as the independent variables (Box 1). Univariate associations between independent variables and the postoperative hematocrit level were analyzed, and variables that were significant at an $\alpha$ level of 0.10 or less were entered into a multivariable linear mixed model, with random site effects to account for clustered data. This multivariable linear mixed model was subjected to stepwise selection to identify a subset of significant model variables with a $P$ value of less than 0.05 in a type III test of fixed effects. The fixed effects were retained only if their addition to the model significantly improved model fit (i.e. decreased the Akaike information criterion by a value consistent with a $P$ value of less than 0.05 for a $\chi^{2}$ distribution with 1 degree of freedom). The retained variables were analyzed for collinearity.

Predicted hematocrit levels were calculated for the randomly selected validation subset, which comprised $20 \%$ of the eligible hysterectomy cases and did not include any data used in the model development subset. The predicted value for each patient was compared with the actual postoperative hematocrit level for that patient, and the difference between the two values was calculated to assess the accuracy of the model for the purposes of prediction.

\section{Results}

Demographic and perioperative data were obtained for 13745 women who underwent hysterectomy at 50 hospitals participating in the MSQC during the study period. After excluding complicated hysterectomies, 9172 (66.7\%) cases were identified as uncomplicated hysterectomies. Of the uncomplicated hysterectomies, 1818 (19.8\%) had no postoperative hematocrit value in the database, and 1420 (15.5\%) had their lowest postoperative hematocrit in the database recorded on a date that was not POD1. As a result, 5934 (64.7\%) uncomplicated hysterectomies were eligible for analysis, 4750 (80.0\%) of which were randomly allocated into a model development dataset and 1184 (20.0\%) to a validation dataset. Three cases were subsequently found to lack preoperative body weight; as a result, the final number in the model development dataset was 4747 .

The subsets were similar in terms of patient age, body mass index, ethnic origin, and smoking status (Table 1). The difference in distribution of route of hysterectomy between subsets reached statistical significance $(P=0.05)$ with a slightly lower proportion of abdominal hysterectomies and greater proportion of vaginal hysterectomies in the model validation subset than in the model development subset.

In the mixed multivariable linear regression, factors associated with a higher POD1 hematocrit included higher weight, higher preoperative hematocrit, and non-vaginal hysterectomy route. Variables associated with a lower POD1 hematocrit value included higher preoperative platelet count, higher EBL, and larger volume of intraoperative crystalloid infusion (Table 2). Analysis of the variables included in the final model revealed no significant collinearity (data not shown).

For purposes of validation, the ability of the model to predict POD1 hematocrit to a value within $1 \%$ to within $5 \%$ points was tested (Table 3). The model predicted the POD1 hematocrit level to $\pm 5 \%$ points for $100 \%, \pm 4 \%$ points for $91.7 \%, \pm 3 \%$ points for $81.2 \%, \pm 2 \%$ points for $62.9 \%$, and $\pm 1 \%$ points for $34.8 \%$ of cases. The squared correlation coefficient $\left(R^{2}\right)$ of the model (calculated for the whole study sample including the validation subset) was 0.53 .

Given the finding that a vaginal hysterectomy route was associated with lower POD1 hematocrit, the change in hematocrit (measured as the difference between POD1 hematocrit and preoperative hematocrit) was analyzed by route of hysterectomy. The mean change in hematocrit was significantly greater for cases of vaginal hysterectomy versus other routes of hysterectomy $(-6.40 \% \pm 2.93 \%$ vs $-5.82 \% \pm 2.92 \%$; $P<0.001)$. By route of hysterectomy, overall mean EBL was highest for abdominal $(201.2 \pm 128.0 \mathrm{~mL})$, followed by vaginal $(141.0 \pm$ $102.2 \mathrm{~mL}$ ), laparoscopic-assisted vaginal hysterectomy (130.5 \pm $105.8 \mathrm{~mL}$ ), and then laparoscopic hysterectomy (91.3 $\pm 78.5 \mathrm{~mL})$. However, vaginal hysterectomy had significantly greater EBL as compared with all other routes $(141.0 \pm 102.2 \mathrm{~mL}$ vs $126.9 \pm 108.4 \mathrm{~mL} ; P<0.001)$.

\section{Discussion}

In the present study, a mathematical model has been developed to predict POD1 hematocrit levels after hysterectomy for benign disease.

Box 1

Variables analyzed within the linear mixed model.

\begin{tabular}{|c|c|c|c|}
\hline Demographic characteristics & Preoperative & Intraoperative & Postoperative \\
\hline $\begin{array}{l}\text { Age }{ }^{a} \text {, ethnic origin }{ }^{a}, \text { body mass index }{ }^{a}, \\
\text { height, weight }{ }^{a} \text {, diabetes, smoking status }{ }^{a} \text {, } \\
\text { ASA class, functional status }\end{array}$ & Hematocrit $^{a}$, platelet count ${ }^{a}$ & $\begin{array}{l}\text { Route of hysterectomy }{ }^{a} \text {, type of anesthesia, } \\
\text { temperature }{ }^{a} \text {, intraoperative crystalloid volume }{ }^{a} \text {, } \\
\text { EBL }^{a} \text {, urine output }{ }^{a} \text {, surgical time }{ }^{a}\end{array}$ & Temperature \\
\hline
\end{tabular}

Abbreviations: ASA, American Society of Anesthesiologists; EBL, estimated blood loss.

${ }^{a}$ Variables independently predictive of postoperative day 1 hematocrit levels when analyzed by bivariate logistic regression. 
Table 1

Demographic characteristics of the model development and validation subsets. ${ }^{\mathrm{a}}$

\begin{tabular}{llll}
\hline Demographics & $\begin{array}{l}\text { Model development subset } \\
(\mathrm{n}=4747)\end{array}$ & $\begin{array}{l}\text { Validation subset } \\
(\mathrm{n}=1184)\end{array}$ & $P$ value $^{\mathrm{b}}$ \\
\hline Age, y & $47.7 \pm 11.3$ & $47.5 \pm 11.1$ & 0.57 \\
BMI & $30.3 \pm 7.0$ & $30.4 \pm 7.0$ & 0.22 \\
Ethnic origin & $3705(78.1)$ & $938(79.2)$ & 0.85 \\
$\quad$ White & $676(14.2)$ & $160(13.5)$ & - \\
Black & $55(1.2)$ & $13(1.1)$ & - \\
Other & $311(6.6)$ & $73(6.2)$ & - \\
$\quad$ Unknown & $1106(23.3)$ & $284(24.0)$ & 0.60 \\
Smoker & & $207(17.5)$ & 0.05 \\
Type of hysterectomy & $954(20.1)$ & $508(42.9)$ & - \\
$\quad$ Abdominal & $2,047(43.1)$ & $213(18.0)$ & - \\
Laparoscopic & $721(15.2)$ & $256(21.6)$ & - \\
Vaginal & $1025(21.6)$ & & \\
LAVH & &
\end{tabular}

Abbreviations: BMI, body mass index (calculated as weight in kilograms divided by the square of height in meters); LAVH, laparoscopic-assisted vaginal hysterectomy.

a Values are given as mean \pm SD or number (percentage).

b By $\chi^{2}$ test for categorical variables and independent $t$ test for continuous variables.

All the perioperative factors tested in the analysis were selected a priori because they have a biological role that plausibly might affect POD1 hematocrit values. Three factors were identified to predict higher POD1 hematocrit: preoperative weight, preoperative hematocrit, and route of hysterectomy other than vaginal. The precedence for including these variables in the model is supported by previous studies.

For example, total blood volume, of which hematocrit represents the percentage of red blood cells, can be calculated on the basis of height and weight, and increases in both correlate with increased blood volume [9]. Thus, women with a higher preoperative weight would have greater overall blood volume such that small shifts in fluid have less impact. Similarly, for any given blood loss, a higher preoperative hematocrit can be expected to correlate with higher POD1 hematocrit, as compared with a lower starting hematocrit value. Lastly, using vaginal hysterectomy as the referent group, all other hysterectomy routes were correlated with higher POD1 hematocrit levels. This finding is probably related to greater intraoperative blood loss with vaginal hysterectomy, as reported in other studies [10-12].

In the present study, EBL was significantly greater among women undergoing vaginal versus non-vaginal hysterectomy and, similarly, there was a greater decrease in hematocrit postoperatively after vaginal hysterectomy. Two randomized controlled trials comparing the route of hysterectomy found that vaginal hysterectomy had greater intraoperative blood loss than did abdominal hysterectomy [10,11]. Although the difference did not reach statistical significance in either study, the sample sizes were small (the largest group had 40 patients), and therefore were probably underpowered to detect this difference. Similarly, Bogani et al. [12] reported significantly greater EBL for vaginal versus laparoscopic hysterectomy for benign indications. The greater

Table 2

Variables and their coefficients in the final multivariable model predicting POD1 hematocrit level after hysterectomy.

\begin{tabular}{lcc}
\hline Variable & Coefficient & $P_{\text {value }}{ }^{\mathrm{a}}$ \\
\hline POD1 hematocrit intercept & 7.2644 & $<0.001$ \\
Preoperative hematocrit (in \% points) & 0.6587 & $<0.001$ \\
Weight (in kg) & 0.03222 & $<0.001$ \\
EBL (in mL) & -0.00652 & $<0.001$ \\
Intraoperative crystalloid (in L) & -0.3303 & $<0.001$ \\
Preoperative platelet count $($ in $\times 1000 / \mu \mathrm{L})$ & -0.00457 & $<0.001$ \\
Laparoscopic, LAV, or abdominal hysterectomy $^{\mathrm{b}}$ & 0.2815 & 0.0055 \\
\hline
\end{tabular}

Abbreviations: POD1, postoperative day 1; EBL, estimated blood loss; LAV, laparoscopicassisted vaginal.

a By type III tests for fixed effects.

b The referent was vaginal hysterectomy.
Table 3

Accuracy of the model in predicting postoperative day 1 hematocrit level after hysterectomy.

\begin{tabular}{lc}
\hline $\begin{array}{l}\text { Accuracy of predicted } \\
\text { hematocrit, \% points }\end{array}$ & $\begin{array}{l}\text { No. (\%) of predicted cases } \\
\text { within range of actual value }\end{array}$ \\
\hline \pm 1 & $412(34.8)$ \\
\pm 2 & $745(62.9)$ \\
\pm 3 & $961(81.2)$ \\
\pm 4 & $1086(91.7)$ \\
\pm 5 & $1184(100.0)$ \\
\hline
\end{tabular}

EBL, and therefore greater drop in postoperative hematocrit, observed during vaginal hysterectomy might be due to initiation of the procedure with a colpotomy (which can subsequently bleed throughout the operation), decreased utilization of electrosurgical coagulation as compared with other routes, limited visualization of blood loss, and increased concomitant procedures (e.g. prolapse or incontinence procedures).

Three variables in the present model were predictive of lower POD1 hematocrit: higher EBL at the time of surgery, greater intraoperative crystalloid volume, and higher preoperative platelet count. Greater EBL at the time of surgery would be expected to lower POD1 hematocrit, as would greater volumes of crystalloid owing to dilutional effects. Although the association between higher preoperative platelet count and lower postoperative hematocrit is not clear, a plausible explanation is that higher preoperative platelet counts may indicate dehydration, and therefore aggressive intraoperative fluid resuscitation might lead to greater dilutional effects.

The present study has several limitations. First, data were limited to what was available in the MSQC database and it was not possible to collect missing data. Second, the analysis was intentionally restricted to benign, uncomplicated hysterectomies with an intraoperative blood loss of $500 \mathrm{~mL}$, which limits the generalizability of the findings. Third, variation between study sites might affect the final model, although the data were analyzed by mixed modeling to account for this effect. Last, the $R^{2}$ and validation outcomes indicate that the model accounts for only approximately $50 \%$ of the variation in POD1 hematocrit. This suggests that there are other factors that affect postoperative blood counts that were not identified in the present study.

The strengths of the present study include the use of a large statewide database representative of various clinical settings. Data in the MSQC database are collected using a validated methodology and by specially trained and dedicated nurse data abstractors. In addition, the use of mixed modeling for statistical analyses controlled for systematic differences among study sites, thereby improving the model's generalizability. Last, the model was validated using $20 \%$ of cases, none of which was used in the initial model development data set.

Although routine measurement of POD1 hematocrit after benign gynecologic procedures is common practice, neither the cost-effectiveness nor the clinical utility of this practice is supported by evidence. Chamsy et al. [13] have estimated that elimination of routine hemoglobin testing after total laparoscopic hysterectomy would save more than US\$2.8 million annually in the USA. Because their study was limited to one route of hysterectomy, healthcare savings would be expected to be much higher if this routine practice were eliminated for all hysterectomies performed for benign indications in the USA. Furthermore, although one unnecessary postoperative hematocrit test might seem innocuous, in actuality it can lead to more costly and more invasive procedures for patients including repeat laboratory testing to validate or refute prior testing, unnecessary imaging studies and radiation exposure, surgical interventions, and delayed hospital discharge. Despite the additional interventions that may be incurred, it has been clearly demonstrated that the practice of routine hematocrit testing after benign gynecologic surgery does not improve outcomes $[3,4]$. Therefore, use of the present model could help to eliminate the practice 
of routine postoperative hematocrit testing after uncomplicated hysterectomy for benign indications, thereby promoting both the practice of evidence-based medicine and good stewardship of resources.

In summary, a model has been developed that might help to limit unnecessary laboratory testing after benign hysterectomy. This model should be used at the completion of surgery to calculate the expected POD1 hematocrit. If the predicted hematocrit is within an acceptable range and the surgeon has no concerns regarding the patient's condition, it would be reasonable to not carry out routine POD1 hematocrit testing. It must be emphasized, however, that this model should be limited to cases of uncomplicated hysterectomy for benign disease among hemodynamically stable patients where there is low suspicion of bleeding complications. Patients who do not meet these criteria should have an evaluation including relevant laboratory assessment as indicated.

At the study institution, use of the present model has prompted reassessment of the need for routine postoperative laboratory tests after uncomplicated benign gynecologic surgery. As a result, standard practice has been changed to review the need for a postoperative hematocrit test immediately after surgery. Taking into account the patient's clinical history and intraoperative factors, clinicians discuss whether management based on vital signs, urine output, and clinical examination will be adequate and, if so, no postoperative hematocrit test is conducted. By incorporating this model into standard practice, postoperative laboratory testing has been reduced among patients undergoing benign gynecologic surgery.

\section{Acknowledgments}

M.B.B. was supported through a National Institute of Child Health and Human Development BIRCWH Career Development Award (\# K12 HD001438). The Michigan Surgical Quality Collaborative is funded by Blue Cross Blue Shield of Michigan and Blue Care Network.

\section{Conflict of interest}

The authors have no conflicts of interest.

\section{References}

[1] Wu JM, Wechter ME, Geller EJ, Nguyen TV, Visco AG. Hysterectomy rates in the United States, 2003. Obstet Gynecol 2007;110(5):1091-5.

[2] Clarke-Pearson DL, Geller EJ. Complications of hysterectomy. Obstet Gynecol 2013; 121(3):654-73.

[3] Kohli N, Mallipeddi PK, Neff JM, Sze EH, Roat TW. Routine hematocrit after elective gynecologic surgery. Obstet Gynecol 2000;95(6 Pt 1):847-50.

[4] Murphy AM, Tunitsky-Bitton E, Krlin RM, Barber MD, Goldman HB. Utility of postoperative laboratory studies after female pelvic reconstructive surgery. Am J Obstet Gynecol 2013;209(4):363.e1-5.

[5] Hannan EL, Samadashvili Z, Lahey SJ, Culliford AT, Higgins RS, Jordan D, et al. Predictors of postoperative hematocrit and association of hematocrit with adverse outcomes for coronary artery bypass graft surgery patients with cardiopulmonary bypass. J Card Surg 2010;25(6):638-46.

[6] Petersen LA, Lindner DS, Kleiber CM, Zimmerman MB, Hinton AT, Yankowitz J. Factors that predict low hematocrit levels in the postpartum patient after vaginal delivery. Am J Obstet Gynecol 2002;186(4):737-44.

[7] Howe C, Paschall C, Panwalkar A, Beal J, Potti A. A model for clinical estimation of perioperative hemorrhage. Clin Appl Thromb Hemost 2003;9(2):131-5.

[8] Campbell Jr DA, Kubus JJ, Henke PK, Hutton M, Englesbe MJ. The Michigan Surgical Quality Collaborative: a legacy of Shukri Khuri. Am J Surg 2009;198(5 Suppl.): S49-55.

[9] Nadler SB, Hidalgo JH, Bloch T. Prediction of blood volume in normal human adults. Surgery 1962;51(2):224-32.

[10] Miskry T, Magos A. Randomized, prospective, double-blind comparison of abdominal and vaginal hysterectomy in women without uterovaginal prolapse. Acta Obstet Gynecol Scand 2003;82(4):351-8.

[11] Ottosen C, Lingman G, Ottosen L. Three methods for hysterectomy: a randomised, prospective study of short term outcome. BJOG 2000;107(11):1380-5.

[12] Bogani G, Cromi A, Uccella S, Serati M, Casarin J, Pinelli C, et al. Laparoscopic versus vaginal hysterectomy for benign indications in women aged 65 years or older: propensity-matched analysis. Menopause 2015;22(1):32-5.

[13] Chamsy DJ, Louie MY, Lum DA, Phelps AL, Mansuria SM. Clinical utility of postoperative hemoglobin level testing following total laparoscopic hysterectomy. Am J Obstet Gynecol 2014;211(3):224.e1-7. 